complement and other innate protective systems against infectious and autoimmunity diseases.

Methods Components of the patient serum complement (CPSC) were registered by immunochemical methods in microplates (variants of functional analyses of isotypes C4A and $\mathrm{C} 4 \mathrm{~B}$, and $\mathrm{C} 1$-inhibitor upon supramolecular assembling on well bottom) and on the blot (preliminary isoelectrophoresis of sera in the plate of polyacrylamide gel). Rabbit and goat polyclonal antibodies against purified CPSC were used. Activity of antibodies-peroxidase conjugates bound to CPSC was detected in the presence of TMB (microplate) or chemiluminescent substrate in a real time [BioChemi System (UVP)].

Results 1.Sera of patients having autoimmune diseases were characterised on the blot by appearance of aggregated $\mathrm{C} 4 \mathrm{~B}$ and C4A within pI 4,0-4,7 compared to less acidic free isotypes. Functional status of isotypes was confirmed in microplate. Absolute amounts of isotypes and their subisotypes as well as ratio of isotypes characterised prognostic-diagnostic patient groups of diseases (SLE, antiphospholipid syndrome, rheumatoid arthritis). Appearance and relative intensities of the system of aggregated isotypes and subisotypes of C4 indicated the presence of disease, its initiation, reached phase of disease and disease character. 2.Similar localization on the blot for the complex $\mathrm{C} 4 \mathrm{~B}$ and C1-inhibitor of patients was registered.

Conclusions Results indicate possible cofunctioining C4B and C1-inhibitor in protection complement network upon development of autoimmune diseases. New mechanisms of cascade protection involving new combinations of CPSC may be revealed. Results open new practical possibilities in diagnostics of early, progressive and chronic autoimmune diseases.

\section{COMPLEMENT LECTIN SYSTEM COFUNCTIONS TO OTHER PROTECTIVE PRO-TEIN SYSTEMS INVOLVING RELATIONSHIPS BETWEEN LECTINS AND GLYCOCONJUGATES AGAINST AUTOIMMUNE AND INFECTIOUS DISEASES}

M Lakhtin, V Lakhtin*, S Andina, S Afanasiev, V Aleshkin. G.N. Gabrichevsky research Institute for Epidemiology and Microbiology, Medical Biotechnology, Moscow, Russia

\subsection{6/lupus-2017-000215.343}

Background and aims Innate protection recognising systems of human organism are important and perspective in respect of investigation autoimmune and infectious diseases. The aim was to evaluate lectin system (LS) of complement and its importance for innate interactome against infectious and autoimmune diseases.

Methods Patient complement components (PCC) of sera were estimated by immunochemical methods in microplates (hybrid plate for $\mathrm{C} 4 \mathrm{~A}$ and $\mathrm{C} 4 \mathrm{~B}$ functionality testing) and on the blot (for PCC separated by isoelectrophoresis). Peroxidase activity was detected using TMB or chemiluminescent substrate (for blot, BioChemi System, UVP).

Results Additional glycoconjugates(GC)-binding PCC were registered.

C1-inh revealed affinity to heparin. Patient (SLE, antiphospholipid syndrome, rheumatoid artritis) subisotypes of $\mathrm{C} 4 \mathrm{~B}$ (up to 5) and C4A (up to 7) were observed as aggregated forms together with GC.
Conclusions 1. Extended complement LS includes MBL, Factor H, C1-inh, CR1, CR2, CR3, C3, C4B, others. 2. Complement serve as a universal communicator among protective systems involving their LS-GC communications. 3. Complement (as mostly advanced innate protection system) possesses structurefunction principles prognostic for any innate recognition systems. 4. There is superLS network in organism. Probiotic LS is important cofunctioning part of it. 5. The data support idea that any protection protein system partially functions involving LS-GC recognition (also for antibodies recognised by Fc-receptors as LS). 6. New prognostic-diagnostic possibilities in investigation of autoimmune and infectious diseases are opened using interactome LS-GC network.

\section{Innate immunity}

\section{LINKING MACROPHAGE MIGRATION INHIBITORY FACTOR AND NLRP3 IN THE PATHOGENESIS OF IL-1 DEPENDENT INFLAMMATORY DISORDERS}

${ }^{1} \mathrm{~T}$ Lang ${ }^{*},{ }^{1} \mathrm{~J}$ Lee, ${ }^{1} \mathrm{~A}$ Pinar, ${ }^{1} \mathrm{H}$ Fan, ${ }^{2} \mathrm{~A}$ Mansell, 'E Morand, ${ }^{1} \mathrm{~J}$ Harris. ${ }^{1}$ Monash University, School of Clinical Sciences at Monash Health, Clayton, Australia; ${ }^{2}$ Hudson Institute of Medical Research, Centre for Innate Immunity and Infectious Diseases, Clayton, Australia

\subsection{6/lupus-2017-000215.344}

Background and Aims Macrophage migration inhibitory factor (MIF) is a mediator of innate immunity and is implicated in the pathogenesis of numerous autoinflammatory disorders. Clinical studies have shown correlations between elevated levels of IL-1 $\beta$ and MIF in serum with disease outcomes of patients with autoimmune disorders. To date, it is unclear whether MIF specifically regulates the expression and secretion of IL-1 family cytokines. Therefore, we aim to characterise mechanisms by which MIF may modulate the secretion of IL1 family cytokines.

Methods The biological activity of MIF in murine bone marrow derived macrophages (BMDM) was inhibited using a small molecule inhibitor, COR123625. Concurrently, BMDM derived from $\mathrm{Mif}^{--}$mice were employed to evaluate the effects of MIF depletion on regulation of IL-1 family cytokines. mRNA expression was analysed by qRTPCR. Protein expression and secretion of IL-1 $\alpha$, IL-1 $\beta$ and IL-18 was assessed by western blot and ELISA.

Results We show that depletion of MIF in macrophages significantly reduced IL-1 cytokine release specifically in response to NLRP3 stimuli, but has no effect on the secretion of TNF$\alpha$ and IL-6. Moreover, diminished IL-1 responses were independent of production of pro-IL-1 $\beta$. Instead, MIF depletion specifically inhibits NLRP3-mediated IL-1 responses as levels were unaffected following activation of AIM2 or NLRC4 inflammasomes.

Conclusions Our findings reveal a novel role for MIF in the modulation of IL-1-dependent inflammatory responses, linking MIF directly to NLRP3 inflammasome activation. This study for the first time implicates a specific role for MIF in the release of IL-1 family cytokines and highlights the potential of targeting MIF in IL-1-dependent pathologies. 